\title{
O nexo entre o consumo de energia primária e o crescimento econômico nos países da América do Sul: uma análise de longo prazo
}

\author{
The nexus between the primary energy consumption and economic \\ growth in South American countries: a long-time span analysis
}

1 Matheus da Costa Koengkan matheuskoen@hotmail.com.br

1 Mestrando em Economia na Universidade da Beira Interior (Covilhã Portugal) ,Pós-graduado em Controladoria e Finanças pela Universidade Federal Fluminense UFF (2015), Graduado em Ciências Contábeis pelo Centro Universitário de Volta Redonda UNIFOA (2013) .Atualmente pesquisador nas áreas da Economia da Energia e Finanças Empresariais na Universidade da Beira Interior.

\section{Resumo}

Este artigo investiga o nexo entre o consumo de energia primária e o crescimento econômico em sete países da América do Sul, no período de 1966-2015. 0 modelo auto-regressivo com desfasamento distribuídos (ARDL) foi utilizado como metodologia. Os resultados das semielasticidades e elasticidades provaram que existe uma relação de feedback entre as variáveis, e que os países estudados possuem uma grande dependência de energia primária, para que seja possível o seu crescimento.

\section{Palavras-chave}

consumo de energia primária; crescimento econômico; América do Sul; ARDL; Relação; Feedback.

\section{Abstract}

This article investigates the nexus between primary energy consumption and economic growth in seven South American countries, from 1966 to 2015. To meet this goal, the Auto-Regressive distributive lag (ARDL) model was used as a methodology. The obtained results from semi-elasticities and elasticities evidentiate that there is a feedback relationship between variables and also shows that all studied countries are highly dependent of primary energy to be able to grow.

\section{Como você deve citar?}

KOENGKAN, Matheus da Costa . O nexo entre o consumo de energia primária e o crescimento econômico nos países da América do Sul: uma análise de longo prazo. Cadernos UniFOA, Volta Redonda, n. 34, p. 63-74, ago. 2017.

\section{Keywords}

Primary energy consumption, Economic growth, South America, ARDL, Relationship, Feedback. 
O nexo entre o consumo de energia primária e o crescimento econômico nos países da América do Sul: uma análise de longo prazo

\section{1.INTRODUÇÃO}

0 estudo da relação entre consumo de energia e crescimento econômico tem recebido ampla atenção na literatura, exceto na abordagem aos países da América do Sul, vindo a revelar-se escassa. Desse modo, a escolha desses países é justificada tanto pela sua ausência nos campos de investigação, como pelo fato dessa região ter registrado um grande consumo de energia nas últimas décadas. Seguindo essa direção, a causalidade entre o consumo de energia e o crescimento econômico é muito importante para a criação de políticas públicas, algo fundamental para o desenvolvimento. Além disso, o uso de energia é um componente importante para o crescimento, e como tal, as políticas de conservação que são orientadas a reduzir o consumo de energia podem ter consequências econômicas negativas, afetando, desse modo, o desenvolvimento econômico de um país.

O objetivo deste estudo é entender o nexo entre o consumo de energia primária e o crescimento econômico nos países da América do Sul. Para que esse objetivo seja atingido, será analisada a relação entre o consumo de energia e o crescimento econômico no período de 1966 a 2015, usando o modelo autorregressivo com desfasamentos distribuídos (ARDL).

Como foi referido anteriormente, essa relação entre consumo de energia e o crescimento económico tem sido amplamente investigada. Por exemplo, alguns estudos empíricos indicam a existência de uma unidirecionalidade vinda do consumo de energia para o crescimento econômico (AHMED e AZAM, 2016; BOUOIYOUR e SELMI, 2014; HAMIT-HAGGAR, 2012), uns indicam a existência de uma única direção de causalidade vindo da atividade econômica para o consumo de energia (AHMED e AZAM, 2016 ; BOUOIYOUR e SELMI, 2014), outros apontam para uma relação de neutralidade, na qual o consumo de energia gera um pouco ou nenhum impacto sobre o crescimento (BOUOIYOUR e SELMI, 2014) e, finalmente, outros apontam para uma relação bidirecional , na qual o consumo de energia e o crescimento são interligados ou vice-versa (ZHAO et al., 2016; AHMED e AZAM , 2016; XIONG et al., 2015).

A relação entre o consumo de energia e o crescimento econômico é inconclusiva, devido aos diversos conflitos sobre os resultados. Baseado neles, foram criadas quatro hipóteses para ajudar atingir o objetivo central do artigo: H1: Hipótese de crescimento; H2: Hipótese de conservação; H3: Hipótese de neutralidade; H4: Hipótese de feedback.

0 artigo está organizado da seguinte forma: A Seção 2 apresenta a revisão da literatura. A Seção 3 apresenta as bases de dados utilizadas e modelo. A Seção 4, os resultados empíricos. A Seção 5, as discussões e, finalmente, as conclusões na Seção 6.

\section{REVISÃO DA LITERATURA}

O nexo entre o consumo de energia e o crescimento econômico tem sido explorado por vários estudos na literatura. A Tabela 1 apresenta o resumo da revisão da literatura com diferentes autores, períodos, metodologias, países, direções de causalidade e hipóteses sobre o tema. 
Tabela 1 - Resumo da revisão da literatura

\begin{tabular}{|c|c|c|c|c|c|}
\hline Autor(es) & Período & Metodologia & País (es) & $\begin{array}{l}\text { Direção (ões) de } \\
\text { causalidade }\end{array}$ & Hipótese(s) \\
\hline $\begin{array}{l}\text { Zhao et al., } \\
\text { (2016) }\end{array}$ & $1995-2014$ & $\begin{array}{l}\text { Painel de } \\
\text { correlações }\end{array}$ & China & Energia Crescimento & Feedback \\
\hline $\begin{array}{l}\text { Ahmed e Azam } \\
\quad(2016)\end{array}$ & $\begin{array}{l}1960-2012 \\
1970-2012\end{array}$ & $\begin{array}{c}\text { Causalidade de } \\
\text { Granger }\end{array}$ & $\begin{array}{l}30 \text { OCDE de alto } \\
\text { rendimento, } 13 \\
\text { de alta renda não } \\
\text { OCDE, } 65 \text { de renda } \\
\text { média e } 11 \text { de } \\
\text { baixa renda. }\end{array}$ & $\begin{array}{c}\text { Energia Crescimento; } \\
\text { Crescimento } \\
\text { Energia; } \\
\text { Energia } \\
\text { Crescimento }\end{array}$ & $\begin{array}{c}\text { Feedback; } \\
\text { Crescimento; } \\
\text { Conservação. }\end{array}$ \\
\hline $\begin{array}{l}\text { Xiong et al., } \\
(2015)\end{array}$ & $1993-2010$ & N. D & Cazaquistão & Energia Crescimento & Feedback \\
\hline $\begin{array}{l}\text { Bouoiyour e } \\
\quad \text { Selmi } \\
(2014)\end{array}$ & $1975-2010$ & N. D & $\begin{array}{l}\text { Algeria, Egito, } \\
\text { Irão, Omã, Arabia } \\
\text { Saudita, Síria, } \\
\text { Estados Árabes } \\
\text { Unidos, Jordânia, } \\
\text { Marrocos, Sudão, } \\
\text { Tunísia, Turquia. }\end{array}$ & $\begin{array}{c}\text { Energia Crescimento; } \\
\text { Crescimento } \\
\text { Energia; } \\
\text { Energia } \\
\text { Crescimento; } \\
\text { Crescimento } \\
\text { Energia. }\end{array}$ & $\begin{array}{c}\text { Feedback; } \\
\text { Crescimento; } \\
\text { Conservação; } \\
\text { Neutralidade. }\end{array}$ \\
\hline $\begin{array}{c}\text { Fuinhas e } \\
\text { Marques (2012) }\end{array}$ & $1965-2009$ & ARDL & $\begin{array}{l}\text { Portugal, Itália, } \\
\text { Grécia, Espanha e } \\
\text { Turquia. }\end{array}$ & Energia Crescimento & Feedback \\
\hline $\begin{array}{l}\text { Hamit-Haggar } \\
(2012)\end{array}$ & $1990-2007$ & $\begin{array}{c}\text { Painel de } \\
\text { Cointegração }\end{array}$ & Canada & $\begin{array}{c}\text { Crescimento } \\
\text { Energia }\end{array}$ & Crescimento \\
\hline $\begin{array}{l}\text { Wang et } \\
\text { al.,(2011) }\end{array}$ & $1995-2007$ & $\begin{array}{c}\text { Painel de } \\
\text { Cointegração }\end{array}$ & China & Energia Crescimento & Feedback \\
\hline $\begin{array}{l}\text { Eggoh et al., } \\
(2011)\end{array}$ & 1970-2006 & $\begin{array}{c}\text { Painel de } \\
\text { Cointegração }\end{array}$ & Países Africanos & Energia Crescimento & Feedback \\
\hline $\begin{array}{c}\text { Acaravci e Özturk } \\
(2010)\end{array}$ & $1970-2005$ & ARDL & $\begin{array}{l}\text { Países da União } \\
\text { Europeia }\end{array}$ & $\begin{array}{c}\text { Crescimento } \\
\text { Energia }\end{array}$ & Crescimento \\
\hline $\begin{array}{l}\text { Apergis e Payne } \\
(2010)\end{array}$ & $1980-2005$ & $\begin{array}{c}\text { Painel de } \\
\text { Cointegração }\end{array}$ & $\begin{array}{c}\text { Países da América } \\
\text { do Sul }\end{array}$ & $\begin{array}{l}\text { Crescimento } \\
\text { Energia }\end{array}$ & Crescimento \\
\hline $\begin{array}{l}\text { Balcilar et } \\
\text { al.,(2010) }\end{array}$ & $1960-2006$ & VAR & Países do G-7 & $\begin{array}{l}\text { Crescimento } \\
\text { Energia }\end{array}$ & Crescimento \\
\hline
\end{tabular}

Notas: N.D significa não disponível; Organização para a Cooperação e Desenvolvimento Econômico (OCDE); Auto-Regressive Distributed Lag (ARDL); Vector Autoregressive (VAR).

Fonte: do autor, 2017.

O uso de diferentes metodologias, países e séries temporais têm levado a diversos resultados, que não chegam a um consenso sobre esse tema. Entretanto, essas conclusões podem ser divididas em quatro tipos de hipóteses:

$\mathrm{H}_{1}$ : Hipótese de crescimento: trata o consumo de energia como um importante complemento no capital e mercado de trabalho bem como no processo de produção, tanto de forma direta como indireta. 0 consumo de energia é um participante significativo no processo de crescimento econômico, no qual o aumento do consumo de energia promoverá o PIB real, enquanto uma política de conservação de energia terá diversos efeitos sobre o crescimento econômico. Contudo os resultados empíricos indicam uma unidirecionalidade vinda do consumo de energia para o crescimento econômico (BARANZINI et al., 2013).

$\mathrm{H}_{2}$ : Hipótese de conservação: a política de conservação de energia não tem efeito de deterioração sobre o crescimento econômico, isto é, o aumento real do PIB aumenta a demanda de energia. A análise 
O nexo entre o consumo de energia primária e o crescimento econômico nos países da América do Sul: uma análise de longo prazo

empírica demonstra uma única direção de causalidade vinda da atividade econômica para o consumo de energia (LEE e CHANG, 2007).

$\mathrm{H}_{3}$ : Hipótese de neutralidade: essa hipótese afirma que apenas uma pequena parte do consumo de energia não causa impacto real no PIB. Além disso, a política de conservação de energia não tem influência desfavorável no crescimento econômico. Os resultados empíricos ilustram que não existe uma relação entre o consumo de energia e o crescimento econômico (KALIMERIS et al., 2014).

$\mathbf{H}_{4}$ : Hipótese de feedback: essa hipótese acredita que o consumo de energia e o crescimento econômico são interligados e complementares. Os resultados empíricos afirmam que existe uma bidirecionalidade de causa entre as duas variáveis (AL-MULALI et al., 2013).

\section{BASE DE DADOS E MODELO}

Esta seção é dividida em duas partes. A primeira descreve o material utilizado e a segunda contém o modelo utilizado no desenvolvimento desta pesquisa.

\subsection{Base de Dados}

0 artigo analisa sete países da América do Sul, a saber: Argentina, Brasil, Chile, Colômbia, Equador, Peru e Venezuela, para um período de 1966-2015. A escolha desses países e a série temporal são justificáveis devido à disponibilidade de dados. Para analisar o nexo entre o consumo de energia e o crescimento econômico, foram empregadas as seguintes variáveis (ver Tabela 2):

Tabela 2 - Variáveis do modelo

\begin{tabular}{llll}
\hline \multicolumn{1}{c}{ Variáveis } & \multicolumn{1}{c}{ Descrição } & \multicolumn{1}{c}{ Fonte } \\
\hline Produto Interno Bruto (PIB) & LY & $\begin{array}{l}\text { PIB em unidades de moeda local a preços } \\
\text { constantes (LCU). }\end{array}$ & World Bank Data (WBD). \\
\hline Consumo de Energia Primária & LEP & $\begin{array}{l}\text { Consumo de Energia Primária onde se } \\
\text { inclui (combustíveis fosseis e renováveis) } \\
\text { em milhões de toneladas equivalentes de } \\
\text { petróleo. }\end{array}$ & $\begin{array}{l}\text { BP statistical review of world } \\
\text { energy. }\end{array}$ \\
\hline
\end{tabular}

Fonte: do autor, 2017.

A escolha das variáveis seguiu os seguintes critérios (a) existência de dados a longo prazo de consumo de energia primária; (b) dados disponíveis a longo prazo. Para controlar a disparidade populacional entre os países, as variáveis foram transformadas em per capita, utilizando-se a população total de todos os países investigados. A utilização da variável LY a preços constantes é justificável, pois permite contornar a influência das taxas de câmbio. Na análise econométrica, foram utilizados os seguintes softwares: Stata 14.0 e EViews 9.5. A estatística descritiva das variáveis é apresentada na Tabela 3. 
Tabela 3. Estatísticas descritivas

\begin{tabular}{c|ccccc}
\hline \multicolumn{5}{c}{ Estatísticas descritivas } \\
\hline & Obs & Média & Std.Dev & Min. & Max. \\
\hline LY & 350 & 10.5064 & 3.1210 & 7.2290 & 16.2150 \\
LEP & 350 & -13.9253 & 0.6413 & -15.7514 & -12.7161 \\
DLY & 343 & 0.0155 & 0.0453 & -0.1780 & 0.1504 \\
DLEP & 343 & 0.0195 & 0.0488 & -0.1628 & 0.2064 \\
\hline
\end{tabular}

Notas: 0 comando sum do Stata foi utilizado para as estatísticas descritivas das variáveis.

Fonte: do autor, 2017.

Em painéis macro, a dependência da secção transversal (CSD) é uma característica muito comum. Na literatura, existem dois tipos de CSD. A primeira é chamada de autocorrelação espacial ou heterogeneidade espacial, definida por (BALTAGI e ANSELIN, 2001). 0 segundo tipo de CSD é a de independência de longo alcance ou de alcance global, como é chamado por Moscone e Tosetti (2009). Ainda nesse contexto, Fuinhas et al., (2015) afirmam que no primeiro tipo de CSD, têm-se em conta a distância entre as crosses, enquanto o segundo tipo não leva em consideração a distância geográfica entre as crosses. Para identificar a características das séries e crosses, bem como a sua ordem de integração, foram aplicados o teste CSD e o teste de raiz unitária de segunda geração, teste CIPS (ver Tabela 4).

Tabela 4 - CDS-Test e CIPS-Test

\begin{tabular}{|c|c|c|c|c|c|c|c|c|}
\hline & \multicolumn{4}{|c|}{$\begin{array}{l}\text { Dependência de Secção Transversal } \\
\text { (CSD-Teste) }\end{array}$} & \multicolumn{4}{|c|}{$\begin{array}{c}2^{\circ} \text { Geração do CIPS-Teste } \\
\text { (Zt-bar) }\end{array}$} \\
\hline & CSD-Tes & & Corr & $\begin{array}{l}\text { Abs } \\
\text { (Corr) }\end{array}$ & Sem trend & & Com tren & \\
\hline LY & 17.64 & $* * *$ & 0.544 & 0.621 & -1.056 & & -1.522 & * \\
\hline LEP & 24.57 & $* * *$ & 0.758 & 0.758 & -0.063 & & 0.531 & \\
\hline DLY & 9.24 & $* * *$ & 0.288 & 0.302 & -6.392 & $* * *$ & -5.730 & $* * *$ \\
\hline DLEP & 3.27 & $* * *$ & 0.102 & 0.146 & -7.402 & $* * *$ & -7.521 & $* * *$ \\
\hline
\end{tabular}

Notas: CSD-teste possui N $(0,1)$ de distribuição. A hipótese nula desse teste: Existência de Dependência transversal. ***, * Denota significância de 1\% e 10\%. O comando utilizado para a realização do CSD-teste foi xtcd. O CIPS-teste tem a seguinte hipótese nula: as series são de ordem I (1); 0 comando utilizando para realização deste teste foi multipurt.

Fonte: do autor, 2017.

A presença de dependência de secção transversal (CSD) foi identificada em todas as variáveis de curto e longo prazo. O teste de raiz unitária de segunda geração - teste CIPS - foi usado (com ou sem trend) com desfasagem (1). A hipótese nula desse teste indica que as séries são de ordem I (1). Os resultados do teste CIPS indicam que as primeiras diferenças das variáveis são de ordem I (1), ou seja, as variáveis são estacionárias e os logaritmos são variáveis não estacionárias I(0). Esses resultados evidenciam a existência de memória de longo prazo entre as variáveis.

Para reforçar a necessidade deste estudo, a Figura 1 evidencia o consumo de energia primária nos países da América do Sul. 


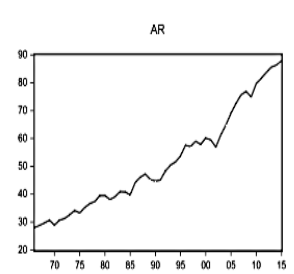

CHL

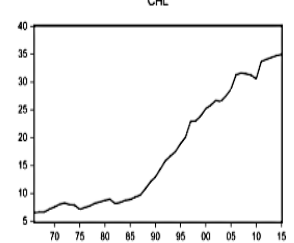

PER

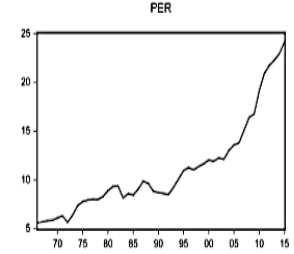

primary
BRA

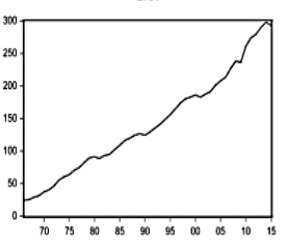

$\mathrm{col}$

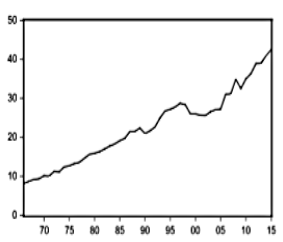

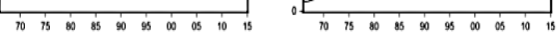

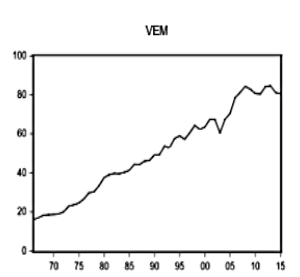

ECU

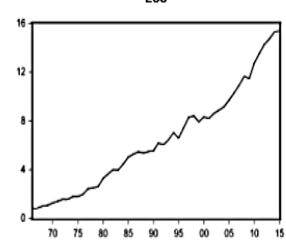

Fonte: do autor, 2017.

Como se pode observar, há existência de choques no consumo energia primária em alguns países e um crescente consumo de energia.

\subsection{Modelo}

O modelo autorregressivo com desfasamentos distribuídos (ARDL) será utilizado, para decompor o efeito total nos efeitos de curto e longo prazo (FUINHAS et al., 2016). O uso recorrente de modelos ARDL no estudo do nexo não se configura como algo novo na literatura. Para identificar os logaritmos naturais e as primeiras diferenças no modelo, foram utilizados os prefixos (L) e (D). A equação geral relacionada às variáveis LY e LEP são as seguintes:

$$
\begin{aligned}
L Y_{t} & =\theta_{0}+\theta_{1} L E P+\mu_{1} t \\
L E P_{t} & =\gamma_{0}+\gamma_{1} L Y+\gamma_{2} t
\end{aligned}
$$

Onde $\theta_{0}$ e $\gamma_{0}$ são os interceptos, $\theta_{1}$ e $\gamma_{1}$ são os coeficientes, e $\mu_{1} t$ e $\gamma_{1} t$ são os termos de distúrbio, assumindo ruído branco e uma distribuição normal. Essas equações fornecem informação sobre as elasticidades a longo prazo. Além disso, assumindo a existência de cointegração entre as 
variáveis e causalidade, a equação (1) e (2) podem ser convertidas em ARDL, como pode ser visto na equação (3) e (4):

$$
\begin{aligned}
L Y_{t} & =\varpi_{0}+\sum_{i=1}^{k} \varpi_{1 i} L Y_{t-i}+\sum_{i=0}^{k} \varpi_{2 i} L E P_{t-i}+\mu_{3} t \\
L E P_{t} & =\varphi_{0}+\sum_{i=1}^{k} \varphi_{1 i} L E P_{t-i}+\sum_{i=0}^{k} \varphi_{2 i} L Y_{t-i}+\mu_{4} t
\end{aligned}
$$

Onde o $\mathcal{K}$ representa o número de desfasagens definido pelo conhecimento empírico das variáveis. Contudo, se as variáveis são cointegradas, o modelo ARDL pode ser transformado em um modelo correcção de erros sem restrições (UECM) (FUINHAS et al., 2016). As equações (3) e (4) serão transformadas em equações (5) e (6), respectivamente, o modelo I e II, que representam o (UECM) equivalente ao modelo ARDL.

$$
\begin{gathered}
D L Y_{t}=\beta_{0}+\sum_{i=1}^{k} \beta_{1 i} D L Y_{t-i}+\sum_{i=0}^{k} \beta_{2 i} D L E P_{t-i}+\beta_{3} L Y_{t-i}+\beta_{4} L E P_{t-i}+\mu_{5} t \\
D L E P_{t}=\sigma_{0}+\sum_{i=1}^{k} \sigma_{1 i} D L E P_{t-i}+\sum_{i=0}^{k} \sigma_{2 i} D L Y_{t-i}+\sigma_{3} L E P_{t-i}+\sigma_{4} L Y_{t-i}+\mu_{6} t
\end{gathered}
$$

A existência de um extenso período de tempo em um macro painel requer a utilização de técnicas que possam lidar com os fenômenos da heterogeneidade e a decomposição de fenômenos de curto e longo prazo. Para identificar o melhor estimador dos modelos, é utilizado o teste Hausman, que identifica a presença de efeitos fixos (FE) ou efeitos aleatórios (RE). No estimador FE, a heterogeneidade é observada no termo constante, sendo este diferente de país para país, enquanto, no estimador $R E$, o coeficiente do termo é independente de uma unidade individual (GREEN, 2000). A hipótese nula do teste Hausman demonstra que o melhor modelo a ser utilizado é o de efeitos aleatórios. Os resultados

do teste Hausman demonstram que o estimador mais adequado é o FE, no qual o $\chi_{3}^{2}$ do Modelo I é (9.08), para o Modelo II (22.09), levando à rejeição da hipótese nula.

\section{RESULTADOS EMPÍRICOS}

A utilização dos dados em painel para o estudo realizado é devido ao elevado número de observações e países. A utilização de macro painéis, além de permitir o controle do fenômeno de heterogeneidade, oferece uma maior gama de informações, variabilidade, graus de liberdade, eficiência e uma menor colinearidade entre as variáveis. Outra vantagem do uso de macro painéis é a capacidade de fazer testes de raiz unitária para ter uma distribuição assintótica normal (BALTAGI, 2005).

Após a realização do teste Hausman, é necessário aplicar dois estimadores: o Mean Group (MG) e o Pooled Mena Group (PMG). O MG, de acordo com Pesaran et al., (1999) é um estimador mais flexível, pois permite que os coeficientes sejam heterogêneos entre vários países. Esse estimador é muito eficaz, quando comparado ao PMG, nas estimações a longo prazo. Já o PMG é também um estimador flexível, porém é bem menor que o MG. Esse estimador executa restrições entre todos os coeficientes a longo prazo, permitindo que a curto prazo exista heterogeneidade nos coeficientes, enquanto a longo prazo 
O nexo entre o consumo de energia primária e o crescimento econômico nos países da América do Sul: uma análise de longo prazo

os coeficientes são homogêneos.Para identificar o melhor estimador (ver Tabela 5), os estimadores MG, PMG e DFE e o teste Hausman foram aplicados.

Tabela 5 - Estimadores heterogêneos e teste Hausman

\begin{tabular}{|c|c|c|c|c|c|c|c|c|c|c|c|c|}
\hline \multirow[b]{4}{*}{ Conctanto } & \multicolumn{6}{|c|}{ Modelo I } & \multicolumn{6}{|c|}{ Modelo II } \\
\hline & \multicolumn{6}{|c|}{ Variável dependente DLY } & \multicolumn{6}{|c|}{ Variável dependente DLEP } \\
\hline & \multicolumn{2}{|c|}{ MG } & \multicolumn{2}{|c|}{ PMG } & \multicolumn{2}{|c|}{ DFE } & \multicolumn{2}{|c|}{ MG } & \multicolumn{2}{|c|}{ PMG } & \multicolumn{2}{|c|}{ DFE } \\
\hline & 1.7213 & $* *$ & 0.2511 & * & 0.6675 & ** & -3.8909 & $\star \star \star *$ & -2.8411 & $\star * *$ & -1.6718 & $* * *$ \\
\hline DLY & & & N. A & & & & 0.5295 & $* * *$ & 0.8713 & $* * *$ & 0.5479 & $* * *$ \\
\hline DLEP & 0.5668 & $\star * *$ & 0.5629 & $* * *$ & 0.5245 & $* * *$ & \multicolumn{6}{|c|}{ N. A } \\
\hline LY & & & N. A & & & & 1.1907 & $* * *$ & 0.5445 & $* * *$ & 0.9404 & $* * *$ \\
\hline LEP & 1.5870 & $* * *$ & -0.1048 & & 0.7510 & $* * *$ & \multicolumn{6}{|c|}{ N. A } \\
\hline ECM & -0.0998 & $* * *$ & -0.0373 & & -0.031 & ** & -0.1587 & $\star * \star *$ & -0.1220 & $* *$ & -0.0707 & $* * *$ \\
\hline $\begin{array}{c}\text { Teste } \\
\text { Hausman }\end{array}$ & \multicolumn{2}{|c|}{ MG vs $P M G$} & \multicolumn{2}{|c|}{ PMG vs DFE } & \multicolumn{2}{|c|}{ MG vs DFE } & \multicolumn{2}{|c|}{ MG vs PMG } & \multicolumn{2}{|c|}{ PMG vs DFE } & \multicolumn{2}{|c|}{ MG vs DFE } \\
\hline$\chi_{4}^{2}$ & N.D & & 0.07 & & 0.00 & $* * *$ & N.D & & 0.00 & $\star * *$ & 0.00 & $* * *$ \\
\hline
\end{tabular}

Notas:***, ** e * denotam significância de 1\%,5\% e 10\%, respectivamente. A hipótese nula do teste Hausman (H0): diferença entre os coeficientes não é sistemática. N.A significa não aplicável e N.D significa não disponível. ECM denota error correction mechanism. 0 comando utilizado no software Stata foi xtpm

Fonte: do autor, 2017.

Os resultados levam à rejeição dos estimadores (MG e PMG), concluindo que o estimador DFE é o mais adequado, rejeitando a presença de heterogeneidade da inclinação dos parâmetros. Para especificar e escolher o melhor estimador, foram aplicados os seguintes testes: (a) teste Wald, para controlar a heterocedasticidade; (b) teste Pesaran, para identificar a dependência transversal dos erros; (c) teste Wooldridge, para aferir a existência de correlação serial; (d) teste Breusch Pagan Langrangian Multiplier, com objetivo de testar a independência entre as crosses e verificar a existência de correlação entre os erros. Os resultados dos testes de especificação do modelo são evidenciados na Tabela 6.

Tabela 6 - Resultados dos testes de especificação

\begin{tabular}{|c|c|c|c|c|}
\hline \multirow{2}{*}{ Teste de Especificação } & \multicolumn{4}{|c|}{ Estatísticas } \\
\hline & Modelo I & & Mod & \\
\hline Teste Wald & $\chi_{7}^{2}=89.64$ & *** & $\chi_{7}^{2}=66.28$ & *** \\
\hline Teste Pesaran & 3.955 & $* * *$ & -1.642 & *** \\
\hline Teste Wooldridge & $F(1,6)=371.984$ & $* * *$ & $F(1,6)=36.203$ & *** \\
\hline Teste Breusch Pagan Langrangian Multiplier & $\chi_{21}^{2}=44.647$ & $* * *$ & $\chi_{21}^{2}=28.558$ & *** \\
\hline
\end{tabular}

Notas: ***,** e * denotam significância de $1 \%, 5 \%$ e $10 \%$, respetivamente. Teste de Wald $\mathrm{H}_{0}$ : sigma(i)^2 para todo o i; teste de Pesaran e Breusch-Pagan LM HO: resíduos não estão correlacionados; teste de Wooldridge HO: não existe autocorrelação de primeira ordem.

Fonte: do autor, 2017.

Os resultados da Tabela 6 rejeitam a hipótese nula do teste Wald, concluindo a existência de heterocedasticidade dos resíduos. 0 teste Pesaran é significante, evidenciando a existência de dependência transversal dos erros nos modelos. Por fim, os testes Wooldridge e Breusch Pagan Langrangian 
Multiplier indicam que os resíduos estão correlacionados e que há autocorrelação de primeira ordem em ambos os modelos.

Após a aplicação dos testes de especificação, é realizado a estimação dos modelos DFE, DFERobusto (DFE-ROB) e Driscoll-Kraay (DFE-DK), nos quais são feitos os cálculos das elasticidades e semielasticidades. As elasticidades de longo prazo não são de leitura direta como as de curto prazo, pois as de curto prazo são iguais aos seus coeficientes. As elasticidades de longo prazo são calculadas pela divisão do coeficiente de cada variável explicativa pelo coeficiente da variável dependente (LY) para o modelo I e (LEP), para o modelo II, são desfasadas, e multiplicadas por (-1).

Tabela 7 - Estimações dos resultados

\begin{tabular}{|c|c|c|c|c|c|c|c|c|}
\hline & \multicolumn{4}{|c|}{ Modelo I } & \multicolumn{4}{|c|}{ Modelo II } \\
\hline & \multicolumn{4}{|c|}{ Variável dependente DLY } & \multicolumn{4}{|c|}{ Variável dependente DLEP } \\
\hline & DFE (I) & & DFE -ROB(II) & $\begin{array}{l}\text { DFE D.K. } \\
\text { (III) }\end{array}$ & DFE (IV) & & $\begin{array}{l}\text { DFE-ROB } \\
\text { (V) }\end{array}$ & $\begin{array}{l}\text { DFE D.-K. } \\
\text { (VI) }\end{array}$ \\
\hline \multirow[t]{2}{*}{ Constante } & 0.6675 & ** & & & -1.6718 & $* * *$ & $* * *$ & $* * *$ \\
\hline & \multicolumn{8}{|c|}{ Curto prazo (semi-elasticidades) } \\
\hline DLY & N. A & & & & 0.5479 & $* * *$ & $* \star *$ & $* * *$ \\
\hline \multirow[t]{2}{*}{ DLEP } & 0.5245 & *** & * & $* * *$ & N. A & & & \\
\hline & \multicolumn{8}{|c|}{ Longo prazo (elasticidades) } \\
\hline $\operatorname{LY}(-1)$ & N. A & & & & 0.9404 & $* * *$ & $* * *$ & $\star \star \star *$ \\
\hline \multirow[t]{2}{*}{$\operatorname{LEP}(-1)$} & 0.7510 & *** & $* * *$ & $\star * * *$ & N. A & & & \\
\hline & \multicolumn{8}{|c|}{ Velocidade de ajustamento } \\
\hline \multirow[t]{2}{*}{ ECM } & -0.0316 & ** & & & -0.0707 & $* * *$ & $* * *$ & $* * *$ \\
\hline & \multicolumn{8}{|c|}{ Estatísticas } \\
\hline $\mathrm{N}$ & 343 & & 343 & 343 & 343 & & 343 & 343 \\
\hline $\mathrm{R} 2$ & 0.2966 & & 0.2966 & 0.2966 & 0.3571 & & 0.3571 & 0.4255 \\
\hline R2_a & 0.0000 & & 0.0000 & N. A & 0.0034 & & 0.0034 & N. A \\
\hline $\mathrm{F}$ & $46.80 * * *$ & & $6.48 * *$ & 24.93 & $61.65 * * *$ & & $51.91 * * *$ & $82.07 * * *$ \\
\hline
\end{tabular}

Notas: ***,**e * denotam significância de $1 \%, 5 \%$ e $10 \%$, respectivamente. Os comandos utilizados no software Stata foram xtreg e xtscc. Na estimação do DK, foi utilizado 1 lag. N.A significa não aplicável.

Fonte: do autor, 2017.

As estimações dos resultados no Modelo I mostram que o consumo de energia primária (LEP) exerce um impacto positivo sobre o crescimento econômico (LY), no qual o aumento de $1 \%$ no consumo de energia tem um impacto de 0.5245 a curto prazo e 0.7510 a longo prazo.

No Modelo II, os resultados evidenciam que o aumento do crescimento econômico (LY) tem uma influência positiva sobre o consumo de energia, no qual o crescimento de 1\% do PIB tem uma influência positiva de 0.5479 a curto prazo e 0.9404 a longo prazo.

\section{DISCUSSÃo}

O objetivo principal deste estudo é identificar qual é a relação entre o consumo de energia e o crescimento econômico, utilizando o modelo ARDL. Os testes iniciais comprovaram a existência de heterocedasticidade, dependência transversal de erros, correlação dos resíduos e a existência de autocorrelação de primeira ordem. 
O nexo entre o consumo de energia primária e o crescimento econômico nos países da América do Sul: uma análise de longo prazo

A análise econométrica deste artigo está focada nos outputs das variáveis LY, LEP e DLY, DLEP. Os resultados das elasticidades evidenciam que o consumo de energia primária no modelo I exerce um impacto positivo sobre o crescimento econômico a curto e longo prazo.

No modelo II, o crescimento econômico tem uma influência positiva sobre o consumo de energia tanto a curto e a longo prazo. As elasticidades mostram que os países da América do Sul são muito dependentes de energia para crescerem, dado que o aumento de $1 \%$ no consumo de energia aumenta de forma significativa o PIB. Esses resultados já eram expectáveis em economias emergentes, pois, como esses países estão em crescimento constante, sempre necessitarão de energia para se desenvolverem. As velocidades de ajustamento dos modelos são negativas e baixas (Modelo I ECM: - 0.0316 e Modelo II ECM: -0.0707), porém estatisticamente significantes, indicando que as economias no estudo são vulneráveis a eventuais choques econômicos, principalmente relacionado ao preço da energia.

Esses resultados estão em concordância com a revisão teórica do artigo, dado que existe uma relação de feedback ou bidirecionalidade entre o consumo de energia e o crescimento econômico ou vice-versa. (ZHAO et al., 2016; XIONG et al., 2015; FUINHAS e MARQUES, 2012).

\section{CONCLUSÕES}

Este artigo aborda a relação entre o consumo de energia primária e o crescimento econômico. De modo a atingir tal propósito, foi utilizado o modelo ARDL com séries temporais estabelecidas entre 1966 a 2015.

Os testes de especificação confirmaram a existência de heterocedasticidade dos resíduos, dependência transversal dos erros e autocorrelação de primeira ordem em ambos os modelos. Essas confirmações asseguram que a aplicação econométrica é adequada.

Os resultados empíricos do Modelo I comprovaram que o consumo de energia primária (LEP) tem uma relação positiva com o crescimento econômico (LY), no qual o aumento de $1 \%$ no consumo de energia tem um impacto positivo de 0.5245 a curto prazo e 0.7510 a longo prazo. No Modelo II, os resultados atestaram que o aumento no crescimento econômico (LY) tem uma relação positiva sobre o consumo de energia primária (LEP), no qual o crescimento de $1 \%$ do PIB tem um impacto positivo de 0.5479 a curto prazo e 0.9404 a longo prazo.

Com base nesses resultados, podemos afirmar que existe uma relação de feedback ou bidirecionalidade, confirmando assim a hipótese $\left(\mathrm{H}_{4}\right)$, de que o consumo de energia e o crescimento econômico estão interligados e complementares. Essa relação bidirecional deve-se ao fato de muitos países da região sul-americana serem dependentes de energia para crescerem.

Essa dependência se deve pelo fato de que alguns países estudados são grandes produtores e exportadores de energia primária, nesse caso, o petróleo, como a Argentina, Brasil, Colômbia, Equador, Peru e Venezuela, e outros apenas importadores, como é o caso do Chile. Além disso, todos os países estudados são emergentes, pois como estão em crescimento constante, sempre necessitarão de energia para se desenvolverem. Todavia, essa dependência é confirmada pelas velocidades de ajustamento dos modelos, que são negativas e baixas. Nesse caso, o modelo I ECM: -0.0316 e o Modelo II ECM: -0.0707 , indicando que os países estudados são vulneráveis a eventuais choques econômicos provocados pela instabilidade de preços da energia ou das commodities, nesse caso, o do petróleo. 
Esses achados são uma oportunidade pertinente para os policy makers criarem políticas e diretrizes destinadas ao desenvolvimento de tecnologias verdes, geração e consumo de energias renováveis, a fim de diversificar a matriz energética, reduzir a dependência dos hidrocarbonetos, impactos ambientas e riscos energéticos.

\section{AGRADECIMENTOS}

É reconhecido o apoio financeiro da NECE - Unidade de Investigação em Ciências Empresariais e Economia, patrocinado pela FCT - Fundação Portuguesa para o Desenvolvimento da Ciência e Tecnologia, Ministério da Educação e Ciência, projeto UID / GES / 04630/2013.

\section{REFERÊNCIAS}

AL-MULALI, U.; LEE, J.Y.M.; MOHAMMED, A.H.; SHEAU-TING, L. Examining the link between energy consumption, carbon dioxide emission, and economic growth in Latin America and the Caribbean. Renewable and Sustainable Energy Reviews, v.26, p.42-48, 2013.doi. 10.1016/j.rser.2013.05.041.

ACARAVCI, A.; ÖZTURK, I. On the relationship between energy consumption, $\mathrm{CO}_{2}$ emissions and economic growth in Europe. Energy, v.35, n.12, p.5412-5420, 2010.doi. 10.1016/j.energy.2010.07.009.

AHMED, M.; AZAM, M. Causal nexus between energy consumption and economic growth for high, middle and low income countries using frequency domain analysis. Renewable and Sustainable Energy Reviews, v.60, p.653-678, 2016.doi 10.1016/j.rser.2015.12.174.

APERGIS, N.; PAYNE, J.E. Energy consumption and growth in South America: evidence from a panel error correction model. Energy Economics, v.32, n.6, p.1421-1426, 2010.doi. 10.1016/j.eneco.2010.04.006.

BALCILAR, M.; OZDEMIR, ZA.; ARSLANTURK,Y. Economic growth and energy consumption causal nexus viewed through a bootstrap rolling window. Energy Economics, v.32, n.6, p.1398-1410, 2010. doi. 10.1016/j.eneco.2010.05.015.

BARANZINI, A.; WEBER, S.; BAREIT, M.; MATHYS, N.A. The causal relationship between energy use and economic growth in Switzerland. Energy Economics, v.36, p.464-470, 2013.doi. 10.1016/j. eneco.2012.09.015.

BALTAGI, B.H. Econometric analysis of panel data. Fourth Edition, Chichester, UK: John Wiley \&Sons, 2008.

BALTAGI, B.H; ANSELIN, L. Spatial econometrics: In a companion to theoretical econometrics. Blackwell: Oxford, p.310-330, 2001.doi.10.1002/9780470996249.ch15.

BOUOIYOUR, J.; SELMI, R. The nexus between electricity consumption and economic growth in MENA countries. Energy Studies Review, v.20, n.2, p.25-41, 2014.doi: 10.15173/esr. v20i2.547.

EGGOH, J.C.; BANGAKE, C.; RAULT, C. Energy consumption and economic growth revisited in African countries. Energy Policy,v.39,n.11,p.7408-7421,2011.doi. 10.1016/j.enpol.2011.09.007. 
O nexo entre o consumo de energia primária e o crescimento econômico nos países da América do Sul: uma análise de longo prazo

FUINHAS, J.A.; MARQUES, A.C.: KOENGKAN, M. Are the renewable energy policies impaction on carbon dioxide emissions? the case of Latin America. Anales de Economia Aplicada XXX, p. 232 - 245,2016. ISSN: 2174-3088.

FUINHAS, J.A.; MARQUES, A.C.; COUTO, A.P. Oil rents economic growth in oil producing countries: Evidence from a macro panel. Economic Change and Restructuring, v.48, n.3, p.257-279, 2015.doi. 10.1007/s10644-015-9170-x.

FUINHAS, J.A.; MARQUES, A.C. Energy consumption and economic growth nexus in Portugal, Italy, Greece, Spain and Turkey: An ARDL bounds test approach (1965-2009). Energy Economics, v.34, n.2, p.511-517, 2012.doi. 10.1016/j.eneco.2011.10.003.

GREENE, W. Econometric analysis. Upper Saddle River, New Jersey: Prentice-Hall,2000.

HAMIT-HAGGAR, M. Greenhouse gas emissions, energy consumption and economic growth: panel cointegration analysis from Canadian industrial sector perspective. Energy Economics, v.34, n.1, p.358-364, 2012.doi. 10.1016/j.eneco.2011.06.005.

KALIMERIS, P.; RICHARDSON, C.; BITHAS, K. A meta-analysis investigation of the direction of the EnergyGDP causal relationship: Implications for the growth-degrowth dialogue. Journal of Cleaner Production, v.67, n.15, p.1-13, 2014.doi. 10.1016/j.jclepro.2013.12.040.

MOSCONE, F.; TOSETTI, E. A review and comparison of tests of cross-section independence in Panels. Journal of Economic Surveys, v.23, n.3, p.528-561, 2009.doi.10.1111/j.1467-6419.2008.00571.x.

PESARAN, M.H.; SHIN, Y.; SMITH, R.P. Pooled mean group estimation of dynamic heterogeneous panels. Journal of American Statistical Association, v.94, n.446, p.621-634,1999. Disponível em: <http://www. jstor.org/stable/2670182>. Acesso em: 26 fev. 2016.

WANG, S.S.; ZHOU, D.Q.; ZHOU.P.; WANG, Q.W. CO2 emissions, energy consumption and economic growth in China: a panel data analysis. Energy Policy, v.39, n.9, p.4870-4875, 2011.doi.10.1016/j. enpol.2011.06.032.

XIONG, C.; YANG, D.; HUO, J.; ZHAO,Y. The relationship between energy consumption and economic growth and the development strategy of a low-carbon economy in Kazakhstan. Journal Arid Land, v.7, n.5, p.706-715, 2015.doi. 10.1007/s40333-015-0132-y.

ZHAO, H.; ZHAO, H.; HAN, X.; HE, Z.; GUO, S. Economic Growth, Electricity Consumption, Labor Force and Capital Input: A More Comprehensive Analysis on North China Using Panel Data. Energy, v.9, p.1-21, 2016.doi. 10.3390/en911089. 